\title{
The Sensitivity of Three-Point Circle Fitting
}

Theodore H. Hopp

U.S. DEPARTMENT OF COMMERCE Technology Administration National Institute of Standards and Technology

Manufacturing Engineering Laboratory Factory Automation Systems Division Gaithersburg, MD 20899

QC

100

.456

H0. 5501

1994 


\title{
NISTIR 5501
}

\section{The Sensitivity of Three-Point Circle Fitting}

\section{Theodore H. Hopp}

\author{
U.S. DEPARTMENT OF COMMERCE \\ Technology Administration \\ National Institute of Standards \\ and Technology \\ Manufacturing Engineering Laboratory \\ Factory Automation Systems Division \\ Gaithersburg, MD 20899
}

September 1994

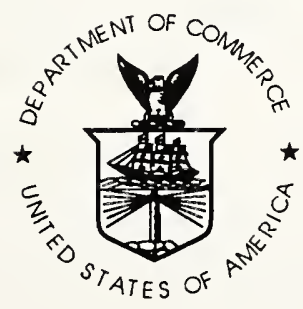

U.S. DEPARTMENT OF COMMERCE Ronald H. Brown, Secretary

TECHNOLOGY ADMINISTRATION Mary L. Good, Under Secretary for Technology

NATIONAL INSTITUTE OF STANDARDS

AND TECHNOLOGY

Arati Prabhakar, Director 


\title{
The Sensitivity of Three-Point Circle Fitting
}

\author{
Theodore H. Hopp \\ National Institute of Standards and Technology \\ Gaithersburg, MD 20899-0001
}

\begin{abstract}
This paper establishes to first order the sensitivity of the center coordinates and radius of a circle through three points in terms of small, random perturbations of those points. This problem arises in the estimation of measurement errors from coordinate measurement systems. Formulas developed herein express the uncertainty of circle parameters as functions of point measurement uncertainty and the arc angle between points. We show that for practical measurement procedures, task uncertainties depend only on the mean and variance of the point measurement errors and are essentially independent of their statistical distribution.
\end{abstract}

Key Words: computational metrology; coordinate measurement machines; coordinate metrology; dimensional metrology; fitting; inspection; measurement uncertainty; substitute geometry; uncertainty

\section{INTRODUCTION}

Coordinate measurement systems are commonly used to inspect the dimensions of manufactured parts. These systems are often used in the following way:

1) measure points on the part surface;

2) fit the points by some means to obtain a "substitute geometry" model of the surface;

3) compare the parameters of the substitute geometry to the product specifications.

In some applications, the last step might be replaced with:

$\left.3^{\prime}\right)$ use the parameters of the substitute geometry to update the process control settings.

For both inspection and process control applications, the quality of the measurement data is an important economic factor. That is, the uncertainty of the fit parameters determines the worth of an inspection. Various standards [1-4] specify methods for determining the uncertainties associated with measuring points on the part surface. Very little has been. published, however, on how these uncertainties propagate through the fitting procedure (Step 2). It is commonly accepted by practitioners in the field that the point measurement uncertainties can have drastically different effects on the end result, depending on the inspection task. A challenge facing industry today is to develop ways of estimating "task-specific uncertainties"-how the point measurement uncertainties affect specific measurements.

A common industry task is measuring an arc of a circle (say, for a fillet or a section of a spherical mirror). Common wisdom is that measuring a small arc (say, $30^{\circ}$ ) can produce unreliable results. Recent work at NIST [5] has used simulation to quantify the uncertainty of the center coordinates and radius of a circle to the arc angle and point uncertainties for this task. Such results allow an inspection planner to determine if a given arc is too small to be measured on a given machine or, conversely, what point uncertainties are needed to measure a given arc. 
Various researchers are advocating the systematic use of simulation to estimate taskspecific uncertainties [6,7]. In this paper, we develop, at least for circles, an analytic approach to this endeavor. This provides an alternative to the computationally intensive simulation approach, and the two approaches can be used to validate one another. Using a simple model of point errors, we develop equations to "derate" the measurement uncertainty of the machine, thereby obtaining task-specific uncertainties for measuring circular arcs.

\section{PROBLEM FORMULATION}

Suppose we wish to inspect a unit circle by measuring three points spaced uniformly over an arc subtending an angle of $2 \theta$, as shown in Figure 1. Typically, we are interested in the size and position of the circle. The point coordinates are $\mathrm{P}_{1}=(-\sin \theta$, $\cos \theta), P_{2}=(0,1)$, and $P_{3}=(\sin \theta, \cos \theta)$. However, these points cannot be measured exactly: each point is perturbed by a small amount from the ideal point on the unit circle. For small perturbations along the circumference, we would expect the resulting fit to remain unchanged. However, radial perturbations will have an effect. So without much loss of accuracy, we can assume that the point perturbations are strictly radial.

The last assumption can be viewed in another way. The perturbation of a point can be decomposed into a radial component and a tangential component. For small perturba-

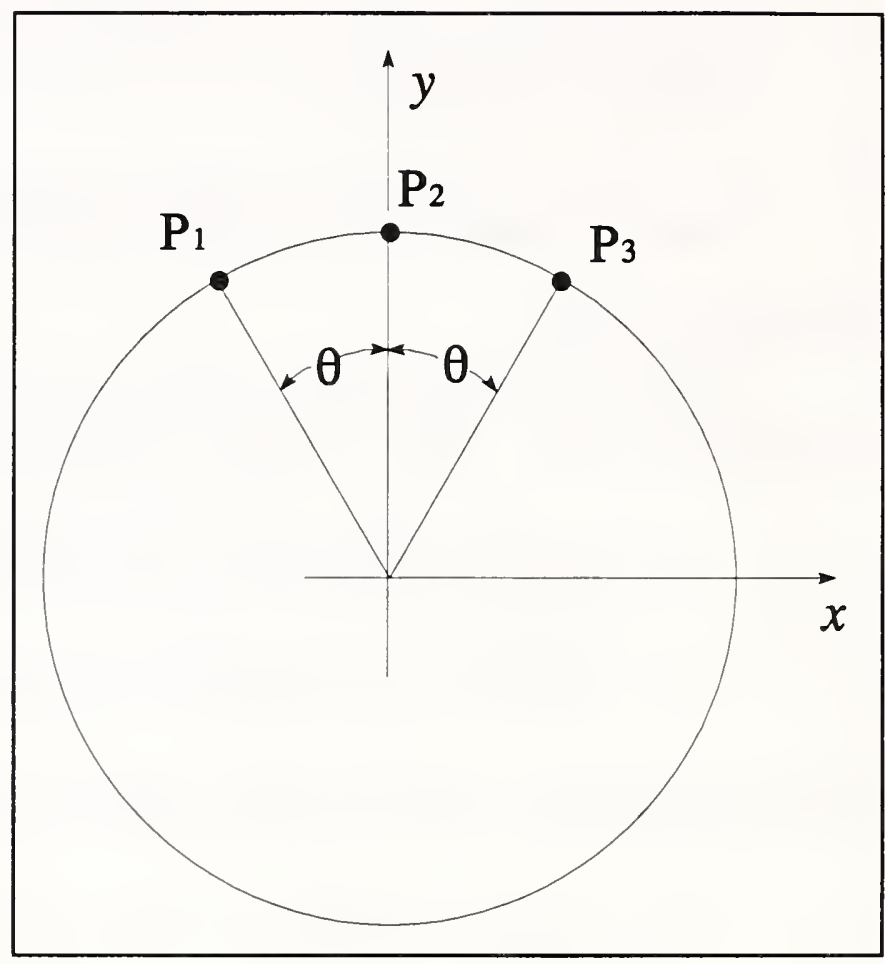

Figure 1. Measuring a circular arc. tions, the tangential component has no effect - the point is still approximately on the circle.

To make things concrete, we will assume that the magnitude of the radial perturbations are strictly random, uncorrelated, and distributed with zero mean and common standard deviation of $\sigma{ }^{1}$ (We might regard the distribution in the radial direction as the marginal distribution of the volumetric error characteristics of the machine.) We will furthermore assume that $\sigma \ll 1$. (Modern coordinate measuring machines typically have point uncertainties on the order of a few microns or less. The smallest features typically measured on such machines are on the order of centimeters. Thus, a practical upper bound for $\sigma$ is $10^{-4}$.)

\footnotetext{
${ }^{1}$ However, this means that our analysis does not apply to the effects of feature deviation from perfect form. That is, our conclusions will be about an imperfect machine measuring a perfect part, not about the influence of part deviations on measurement results. In practice, perturbations are strictly random and uncorrelated only when the measurements extend over a small fraction of the working volume of the machine. The perturbations will have zero mean if the systematic errors of the coordinate measuring machine have been eliminated from the point coordinates. This is the case for many modern, computer-controlled systems. All the assumptions can be tested for a particular machine.
} 
Let the magnitude of the perturbations be $u_{i}, i=1,2,3$. For convenience, define $\gamma_{i}=1+u_{i}$. Then we are fitting a circle to three points with coordinates $\gamma_{i} P_{i}, i=1,2,3$. Since the points are random variables, the center coordinates and radius of the circle going through the three points are also random variables. Our problem is to determine the mean and standard deviation for these quantities. (If we were to assume some particular distribution for the $u_{i}$, we could-in principle-find the distributions of the center coordinates and radius.)

Many results in this paper rely on two results from probability theory. The first is that the mean of the sum of random variables is the sum of their means. (That is, the operations of addition and taking expectation can be interchanged freely.) The second result is the following theorem, the proof of which can be found in many introductory books on probability and statistics (for example, [8]):

Theorem Let $\left\{X_{1}, \ldots, X_{n}\right\}$ be $n$ uncorrelated random variables with means $m_{i}$ and variances $\sigma_{i}{ }^{2}$, and let $Z=H\left(X_{1}, \ldots, X_{n}\right)$. Then (assuming the necessary derivatives of $H$ exist), the mean and variance of $Z$ are approximately

$$
\begin{gathered}
m_{Z} \approx H\left(m_{1}, . ., m_{n}\right)+\frac{1}{2} \sum_{i=1}^{n} \frac{\partial^{2} H}{\partial x_{i}^{2}} \sigma_{i}^{2} \\
\sigma_{Z}^{2} \approx \sum_{i=1}^{n}\left(\frac{\partial H}{\partial x_{i}}\right)^{2} \sigma_{i}^{2}
\end{gathered}
$$

where all the partial derivatives are evaluated at the point $\left(m_{1}, \ldots, m_{n}\right)$.

The proof involves expanding $H$ in a Taylor series about the point $\left(m_{1}, \ldots, m_{n}\right)$ to one or two terms, discarding the remainder, and taking the expectation and variance of both sides. (When higher derivatives are zero, the expressions are exact.)

As special cases of the Theorem, we have the following relationships, which will be used frequently in what follows:

- The mean of $a+X$ is $a+m$

- The mean of $a X$ is $a m$

- The mean of $X^{2}$ is $m^{2}+\sigma^{2}$

- The variance of $a+X$ is $\sigma^{2}$

- The variance of $a X$ is $a^{2} \sigma^{2}$

- If $X_{1}$ and $X_{2}$ are uncorrelated, the variance of $X_{1}+X_{2}$ is $\sigma_{1}{ }^{2}+\sigma_{2}{ }^{2}$

All of the above relationships are exact, since the associated Taylor series terminate.

\section{SOLUTION OF THE CIRCLE EQUATION}

We start by representing the circle in standard form: $x^{2}+y^{2}+a x+b y+c=0$. This corresponds to a circle centered at $(-a / 2,-b / 2)$ with radius $r=1 / 2\left(a^{2}+b^{2}-4 c\right)^{1 / 2}$. Substituting each of the point 
coordinates in turn, we obtain three equations in the unknowns $a, b$, and $c$, which can be written in matrix form as:

$$
\left(\begin{array}{ccc}
-\gamma_{1} \sin \theta & \gamma_{1} \cos \theta & 1 \\
0 & \gamma_{2} & 1 \\
\gamma_{3} \sin \theta & \gamma_{3} \cos \theta & 1
\end{array}\right)\left(\begin{array}{l}
a \\
b \\
c
\end{array}\right)=\left(\begin{array}{l}
-\gamma_{1}^{2} \\
-\gamma_{2}^{2} \\
-\gamma_{3}^{2}
\end{array}\right)
$$

This can be solved using Cramer's rule. The denominator is the determinant of the coefficient matrix:

$$
D=\left|\begin{array}{ccc}
-\gamma_{1} \sin \theta & \gamma_{1} \cos \theta & 1 \\
0 & \gamma_{2} & 1 \\
\gamma_{3} \sin \theta & \gamma_{3} \cos \theta & 1
\end{array}\right|=-\sin \theta\left(\gamma_{1} \gamma_{2}+\gamma_{2} \gamma_{3}-2 \gamma_{1} \gamma_{3} \cos \theta\right)
$$

The $x$ coordinate of the center, $x_{c}$, is determined by coefficient $a$, which in turn is given by

$$
\begin{aligned}
a=\left|\begin{array}{ccc}
-\gamma_{1}^{2} & \gamma_{1} \cos \theta & 1 \\
-\gamma_{2}^{2} & \gamma_{2} & 1 \\
-\gamma_{3}^{2} & \gamma_{3} \cos \theta & 1
\end{array}\right| & =\left(\frac{\gamma_{1}-\gamma_{3}}{\sin \theta}\right)\left(\frac{\gamma_{1} \gamma_{2}+\gamma_{2} \gamma_{3}-\left(\gamma_{1} \gamma_{3}+\gamma_{2}^{2}\right) \cos \theta}{\gamma_{1} \gamma_{2}+\gamma_{2} \gamma_{3}-2 \gamma_{1} \gamma_{3} \cos \theta}\right) \\
& =\left(\frac{\gamma_{1}-\gamma_{3}}{\sin \theta}\right)\left(1+\frac{\left(\gamma_{1} \gamma_{3}-\gamma_{2}^{2}\right) \cos \theta}{\gamma_{1} \gamma_{2}+\gamma_{2} \gamma_{3}-2 \gamma_{1} \gamma_{3} \cos \theta}\right)
\end{aligned}
$$

For small $u_{i}$, the second factor is approximately 1. (The approximation is exact when $\theta=90^{\circ}$.) Thus,

$$
a \approx \frac{\gamma_{1}-\gamma_{3}}{\sin \theta}=\frac{u_{1}-u_{3}}{\sin \theta}
$$

Then $x_{c}$ is 


$$
x_{c}=-\frac{a}{2} \approx \frac{u_{3}-u_{1}}{2 \sin \theta}
$$

Thus, $x_{c}$ has zero mean and standard deviation $\sigma_{x}=\sigma /(\sqrt{2} \sin \theta)$. We will call the ratio $\sigma_{x} / \sigma$ the sensitivity of $x_{c}$. It represents how much more uncertainty there is in $x_{c}$ than there is in the individual measured points.

A plot of the $x$ coordinate sensitivity is shown in Figure 2. The machine's point uncertainty is doubled when measuring arcs of $\theta=20^{\circ}\left(40^{\circ}\right.$ total arc) and continues to grow as $\theta$ gets smaller. The sensitivity of the $x$ coordinate also grows as $\theta$ approaches $180^{\circ}$, corresponding to the indeterminacy of $x$ when the circle is measured using opposing points along the $y$ axis. Note that the uncertainty of $x$ is at a minimum when $\theta=90^{\circ}$, where it has the value $1 / \sqrt{2}$. As we shall see later, this does not represent the minimum in the uncertainty of the center, which also depends on the uncertainty of the $y$ coordinate.

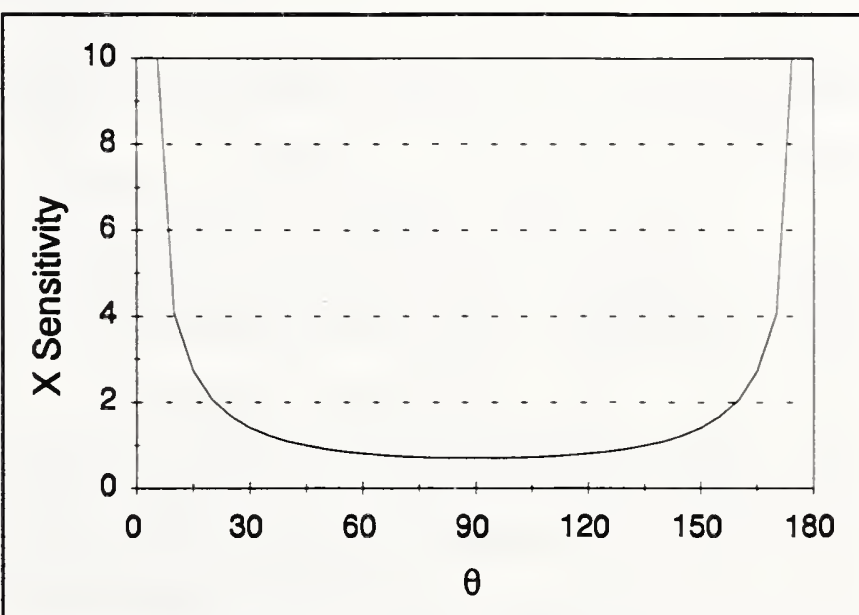

Figure 2 Plot of $x$ center coordinate sensitivity against arc angle between points.

The $y$ coordinate of the center, $y_{c}$, is determined by coefficient $b$, which is given by

$$
b=\frac{\left|\begin{array}{ccc}
-\gamma_{1} \sin \theta & -\gamma_{1}^{2} & 1 \\
0 & -\gamma_{2}^{2} & 1 \\
\gamma_{3} \sin \theta & -\gamma_{3}^{2} & 1
\end{array}\right|}{D}=\frac{\left(\gamma_{1} \gamma_{3}-\gamma_{2}^{2}\right)\left(\gamma_{1}+\gamma_{3}\right)}{\gamma_{1} \gamma_{2}+\gamma_{2} \gamma_{3}-2 \gamma_{1} \gamma_{3} \cos \theta}
$$

For small $u_{i}, b$ is approximately

$$
b \approx \frac{2\left(u_{1}-2 u_{2}+u_{3}\right)}{2(1-\cos \theta)-\left(u_{1}-2 u_{2}+u_{3}\right)}=\frac{u_{1}-2 u_{2}+u_{3}}{1-\cos \theta}\left[\frac{1}{1-\frac{u_{1}-2 u_{2}+u_{3}}{2(1-\cos \theta)}}\right]
$$

This approximation is independent of the choice of $\theta$. If $\theta$ is not too small (i.e., $1-\cos \theta » \sigma$ ) the factor in brackets is about 1 , and we have 


$$
b \approx \frac{u_{1}-2 u_{2}+u_{3}}{1-\cos \theta}
$$

Then $y_{c}$ is

$$
y_{c}=-\frac{b}{2} \approx-\frac{u_{1}-2 u_{2}+u_{3}}{2(1-\cos \theta)}
$$

The numerator is a random variable with zero mean and variance $6 \sigma^{2}$. Thus, $y_{c}$ has zero mean and standard deviation $\sigma_{y} \approx \sqrt{3 / 2} \sigma /(1-\cos \theta)$.

A plot of the $y$ coordinate sensitivity is shown in Figure 3. As with the $x$ coordinate, the uncertainty in $y_{c}$ grows dramatically for small $\theta$. The $y$ coordinate uncertainty is twice the machine point uncertainty when measuring arcs of about $134^{\circ}$ (as compared to $40^{\circ}$ for the $x$ coordinate); in measuring a $40^{\circ}$ total arc $\left(\theta=20^{\circ}\right)$, the $y$ coordinate uncertainty is over 20 times the point uncertainty.

The individual $x$ and $y$ coordinates of the center are of limited interest in metrology applications. (They may be used, for instance, in checking bidirectional position tolerances.) A much more common quantity is the Euclidean distance of the measured center from its specified position. This sug-

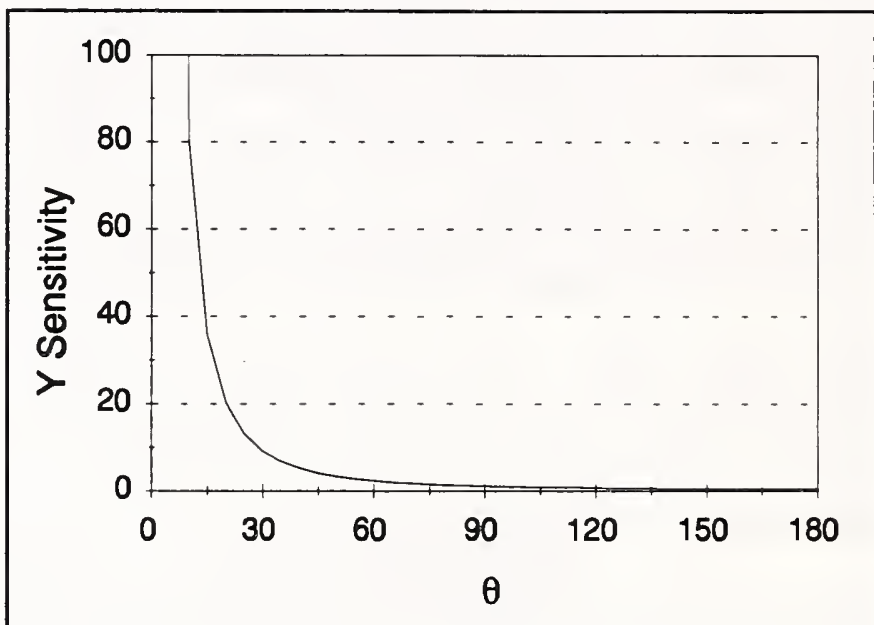

Figure 3 Plot of $y$ center coordinate sensitivity against arc angle between points. (Note that the vertical axis is ten times that of Figure 2.) gests we study the quantity

$$
d=\sqrt{x_{c}^{2}+y_{c}^{2}} \approx \sqrt{\frac{\left(u_{3}-u_{1}\right)^{2}}{4 \sin ^{2} \theta}+\frac{\left(u_{1}-2 u_{2}+u_{3}\right)^{2}}{4(1-\cos \theta)^{2}}}
$$

The expected value of $d$ represents the expected shift of the fit circle from its true center due to point measurement uncertainties. The analysis is complicated by the presence of the square root. We can derive an expression for the expected value of $d^{2}$ as follows. For convenience, let $\alpha=\left(u_{3}-u_{1}\right)^{2}$ and $\beta=\left(u_{1}-2 u_{2}+u_{3}\right)^{2}$. Then the expected values of $\alpha$ and $\beta$ are $2 \sigma^{2}$ and $6 \sigma^{2}$, respectively and 


$$
\mathrm{E}\left(d^{2}\right) \approx \sigma^{2} \frac{2+\cos \theta}{\sin ^{2} \theta(1-\cos \theta)}
$$

A plot of $\sqrt{\mathrm{E}\left(d^{2}\right)} / \sigma$ is shown in Figure 4 . The minimum occurs at $\theta=120^{\circ}$, corresponding to equally spacing the points around the circle. This result is satisfying, because it corresponds to an intuitive sense of how to get the most accurate measurement of a circle center.

Unfortunately, a knowledge of $\mathrm{E}\left(d^{2}\right)$ does not tell us too much about the distribution of $d$. For instance, we do not know the variance of $d^{2}$, which requires knowledge of the fourth moments of the $u_{i}$. If we assumed particular distributions for the $u_{i}$, it would be fairly straightforward to calculate the distribution of $d$.

Nevertheless, we can assume that the

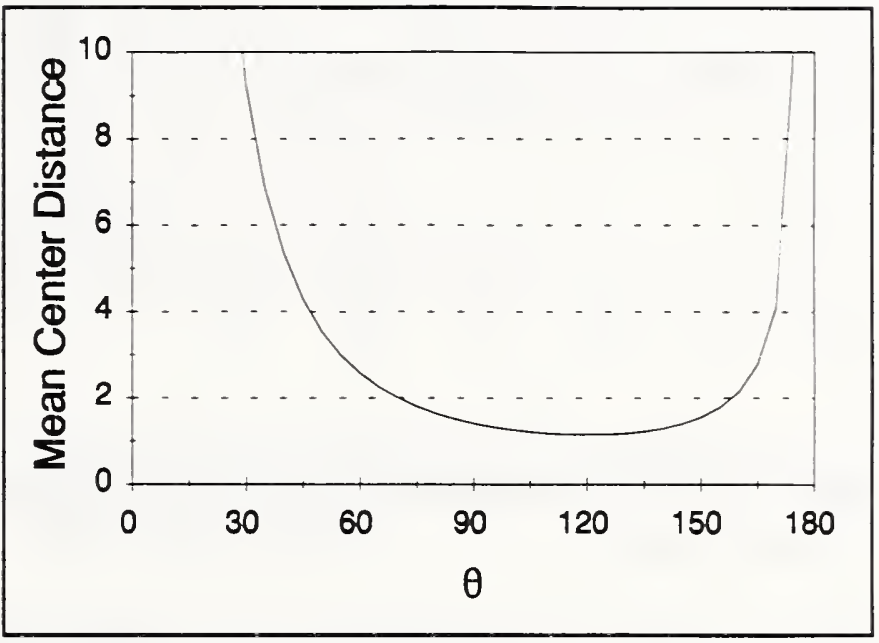

Figure 4 Plot of the expected Euclidean distance of the center from the origin against arc angle between points.

expected value of $d$ will be on the order of the square root of the expected value of $d^{2}$. The expected value of $d$ represents the uncertainty in determining the circle center. As such, it can be used as an estimate of measurement uncertainty in center position in the Euclidean sense. In

this sense, the expression $\sqrt{\mathrm{E}\left(d^{2}\right)} / \sigma$ is analogous to the expressions for the sensitivity of the $x$ and $y$ coordinates developed above.

We now turn to the last characteristic commonly measured for circles: the radius. The radius depends on coefficient $c$ as well as $a$ and $b$. Coefficient $c$ is given by

$$
c=\frac{\left|\begin{array}{ccc}
-\gamma_{1} \sin \theta & \gamma_{1} \cos \theta & -\gamma_{1}^{2} \\
0 & \gamma_{2} & -\gamma_{2}^{2} \\
\gamma_{3} \sin \theta & \gamma_{3} \cos \theta & -\gamma_{3}^{2}
\end{array}\right|}{D}=\frac{-\gamma_{1} \gamma_{2} \gamma_{3}\left(\gamma_{1}+\gamma_{3}-2 \gamma_{2} \cos \theta\right)}{\gamma_{1} \gamma_{2}+\gamma_{2} \gamma_{3}-2 \gamma_{1} \gamma_{3} \cos \theta}
$$

This can be written 


$$
c=-\gamma_{1} \gamma_{3}-\frac{2 \gamma_{1} \gamma_{3}\left(\gamma_{1} \gamma_{3}-\gamma_{2}^{2}\right) \cos \theta}{\gamma_{1} \gamma_{2}+\gamma_{2} \gamma_{3}-2 \gamma_{1} \gamma_{3} \cos \theta}
$$

For small $u_{i}, c$ is approximately

$$
c \approx-\left[1+u_{1}+u_{3}+\frac{\left(u_{1}-2 u_{2}+u_{3}\right) \cos \theta}{1-\cos \theta}\left[\frac{1}{1-\frac{u_{1}-2 u_{2}+u_{3}}{2(1-\cos \theta)}}\right]\right]
$$

and, as with the expression for $b$, when $\theta$ is not too small the expression in the inner brackets is about 1 and

$$
c \approx-\left[1+\frac{u_{1}-2 u_{2} \cos \theta+u_{3}}{1-\cos \theta}\right]
$$

As mentioned above, the circle radius $r$ is given by $r=\frac{1}{2} \sqrt{a^{2}+b^{2}-4 c}$. Observe that $a^{2}$ and $b^{2}$ are on the order of $\sigma^{2}$, while $-c$ is of the form $1+\varepsilon$, where $\varepsilon$ is on the order of $\sigma$. Thus, the radius reduces to

$$
r \approx \sqrt{-c} \approx 1+\frac{\varepsilon}{2}=1+\frac{u_{1}-2 u_{2} \cos \theta+u_{3}}{2(1-\cos \theta)}
$$

Thus, $r$ is distributed with a mean of 1 and standard deviation

$$
\sigma_{r} \approx \sigma \sqrt{\frac{1+2 \cos ^{2} \theta}{2(1-\cos \theta)^{2}}}
$$

A plot of the sensitivity of $r$ as a function of $\theta$ is shown in Figure 5. As with determining the center position, this curve has a minimum at $\theta=120^{\circ}$, where it has a value of $1 / \sqrt{ } 3$. The radius sensitivity becomes less than one at $\cos (\theta)=0.25\left(\theta \approx 75.5^{\circ}\right.$, or a total measured arc of $\left.151^{\circ}\right)$. Although the center position sensitivity rises dramatically as $\theta$ increases past $120^{\circ}$ (because of the rising sensitivity of the $x$ coordinate), the radius remains relatively insensitive for increasing $\theta$. Thus, even for an opposed-point measurement $\left(\theta=180^{\circ}\right)$, the radius sensitivity is only about 0.6 . 


\section{SUMMARY}

We have developed equations for the uncertainty of various circle characteristics when they are determined by measuring three points using a coordinate measuring system with known point measurement uncertainties. Using the angle between points as a parameter, we developed equations and plots that relate the point measurement uncertainty to uncertainty in measuring the center $x$ coordinate, center $y$ coordinate, Euclidean distance of the center from the origin, and circle radius. These task-specific uncertainties depend on the mean and variance of the point measurement errors, but are independent of the

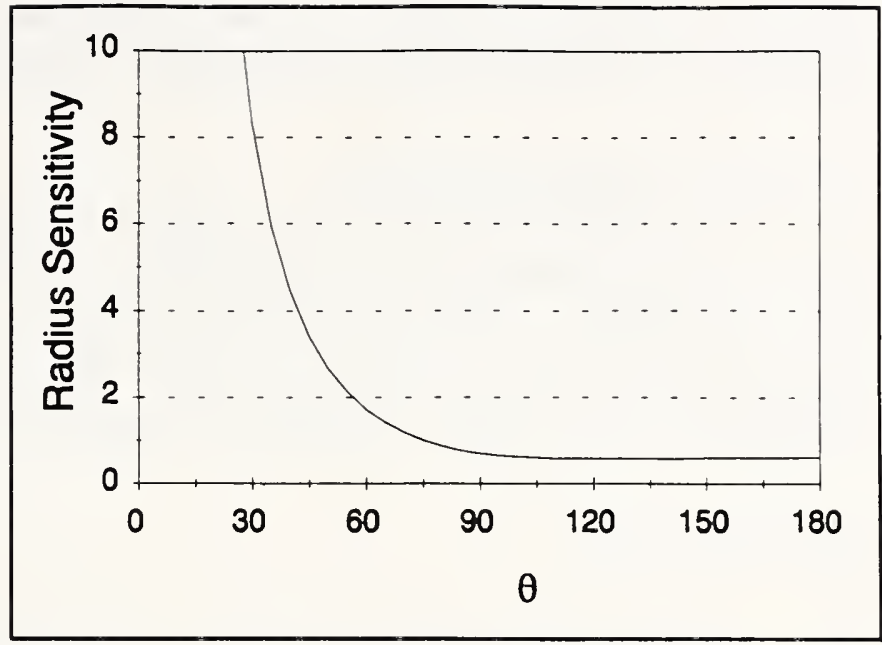

Figure 5 Plot of radius sensitivity against arc angle between points. distributional form of the point errors.

The results developed here apply to measuring a circle with perfect form. (They might apply when measuring a calibration artifact, for instance.) They say nothing about how to measure a part with significant form errors. For one thing, using three points to measure a circle is very poor policy-many processes for making circles (rather, holes and shafts) generate form errors with three-fold symmetry, and three-fold symmetry is the last thing one wants in the surface sampling.

In one sense, our results merely confirm what practitioners have always known. The optimal angle between points-the angle that minimizes the uncertainty of the measured quantity -is $120^{\circ}$ for the radius and the Euclidean center position, and opposed-point measurement for determining a single center coordinate ( $x$ or $y$ ).

The main value of our results is that it provides quantitative methods for estimating taskspecific uncertainties. Even when an inspection planner has the luxury of placing the measurement points in an optimal position, there have been few methods available for estimating the resulting uncertainty. Beyond that, one is often forced to measure a limited arc of a circle. (That may be all there is of the part!) In such cases, one can expect the uncertainty of measured quantities to behave according to the curves presented here.

\section{FUTURE WORK}

One would expect the uncertainty of circle characteristics to decrease with increasing number of points. The results herein will have to be extended to provide guidance as to what to expect with more than three points. More generally, the analysis should be extended to realistic sampling plans and fitting procedures (e.g., many equally-spaced points fit using orthogonal distance regression). This work remains to be done, but we can outline the necessary elements based on our experience with the NIST Algorithm Testing System [9]. To start, we should think of the points as defining a vector of residuals for any particular fit. The proper tool to examining the sensitivity of the solution to small perturbations in the data is the condition number of the Jacobian of this residual vector. By parameterizing the Jacobian by the angle between points, we should be able to develop general expressions for "derating" the machine 
points uncertainty in terms of total measured arc and number of points. The analysis can likewise be extended to other geometries. These extensions will be the subject of future research.

\section{ACKNOWLEDGEMENTS}

The motivation for this work was a problem being studied by Steve Phillips of the Precision Engineering Division of NIST. I wish to thank him for several valuable discussions and comparisons with his simulation results. I would also like to thank Mark Levenson of the Statistical Engineering Division of NIST for his thoughtful review of the paper. This work was supported by the NIST Competence Project on Computational Metrology.

\section{REFERENCES}

[1] Methods for the Performance Evaluation of Coordinate Measuring Machines, ASME B89.1.12-1990, American Society of Mechanical Engineers, New York, NY, 1990.

[2] Coordinate Measuring Machines: Part 2: Methods for Verifying Performance, BS 6808, British Standards Institute, London, 1987.

[3] Accuracy of Coordinate Measuring Machines, Characteristic Parameters and Their Checking, VDI/VDE 2617, Germany.

[4] Test Code for Accuracy of Coordinate Measuring Machines, JIS B7440, Japanese Standards Association, Tokyo, 1987.

[5] Phillips, S., "Performance Evaluations," in J. Bosch (ed.), Coordinate Measuring Machines and Systems, Marcel-Dekker, NY, to appear.

[6] Kunzmann, H., E. Trapet, and F. Wäldele, "Concept for the Traceability of Measurements with Coordinate Measuring Machines," Proc. 7th Int. Prec. Eng. Seminar, Kobe, May 1721, 1993, pp. 1-14.

[7] WECC Technical Guideline, "Coordinate Measuring Machine Calibration," Western European Calibration Cooperation, Oct. 20, 1993 (draft in review).

[8] P. L. Meyer, Introductory Probability and Statistical Applications, Addison-Wesley, Reading, MA, 1970.

[9] Diaz, C. and T. Hopp, "Testing of Coordinate Measuring System Software," Proc. 1993 ASQC Meas. Qual. Conf., Gaithersburg, MD, October 27-29, 1993. 
-

. 


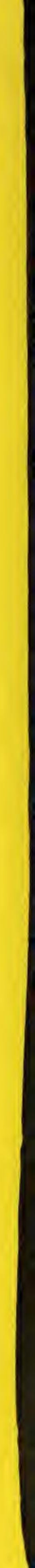

